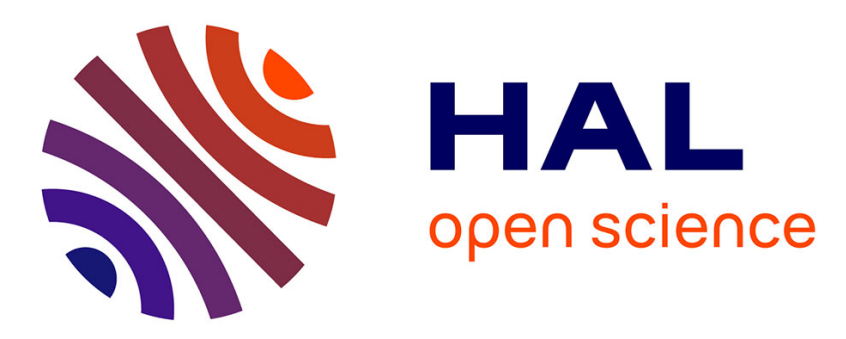

\title{
Influence of the trajectory planning on the accuracy of the orthoglide 5-axis
}

\author{
Ranjan Jha, Damien Chablat, Fabrice Rouillier, Guillaume Moroz
}

\section{To cite this version:}

Ranjan Jha, Damien Chablat, Fabrice Rouillier, Guillaume Moroz. Influence of the trajectory planning on the accuracy of the orthoglide 5-axis. ASME International Design Engineering Technical Conference and the Computer and Information in Engineering Conference (IDETC/CIE), Aug 2016, Charlotte, NC, United States. hal-01309190

\section{HAL Id: hal-01309190 https://hal.science/hal-01309190}

Submitted on 29 Apr 2016

HAL is a multi-disciplinary open access archive for the deposit and dissemination of scientific research documents, whether they are published or not. The documents may come from teaching and research institutions in France or abroad, or from public or private research centers.
L'archive ouverte pluridisciplinaire HAL, est destinée au dépôt et à la diffusion de documents scientifiques de niveau recherche, publiés ou non, émanant des établissements d'enseignement et de recherche français ou étrangers, des laboratoires publics ou privés. 


\title{
Influence of the trajectory planning on the accuracy of the Orthoglide 5 -axis
}

\author{
R. Jha ${ }^{1}$, D. Chablat ${ }^{1}$, F. Rouillier ${ }^{2}$, G. Moroz ${ }^{3}$ \\ ${ }^{1}$ Institut de Recherche en Communications et Cybernétique de Nantes \\ (UMR CNRS 6597), Nantes, France \\ Ranjan.Jha@irccyn.ec-nantes.fr, Damien.Chablat@irccyn.ec-nantes.fr \\ ${ }^{2}$ INRIA Paris-Rocquencourt, Institut de Mathématiques de Jussieu \\ (UMR CNRS 7586), Paris, France \\ ${ }^{3}$ INRIA Nancy-Grand Est, \\ Nancy, France \\ Guillaume.Moroz@inria.fr
}

April 29, 2016

\begin{abstract}
Usually, the accuracy of parallel manipulators depends on the architecture of the robot, the design parameters, the trajectory planning and the location of the path in the workspace. This paper reports the influence of static and dynamic parameters in computing the error in the pose associated with the trajectory planning made and analyzed with the Orthoglide 5-axis. An error model is proposed based on the joint parameters (velocity and acceleration) and experimental data coming from the Orthoglide 5-axis. Newton and Gröbner based elimination methods are used to project the joint error in the workspace to check the accuracy/error in the Cartesian space. For the analysis, five similar trajectories with different locations inside the workspace are defined using fifth order polynomial equation for the trajectory planning. It is shown that the accuracy of the robot depends on the location of the path as well as the starting and the ending posture of the manipulator due to the acceleration parameters.
\end{abstract}

\section{INTRODUCTION}

The accuracy to attain the exact pose for the parallel manipulator for a given trajectory depends on the static and dynamic parameters associated with the manipulator. Due to the better dynamic properties, high loadcarrying capacity, high accuracy and stiffness, closed loop mechanisms are best suited for the medical robotics, highprecision and machine tool design applications []. Number of links and passive joints in the closed loop mechanism reduces the accuracy of the manipulator. There are different factors which affects the accuracy of the manipulator, some of them are geometrical deviation of the machine parts during their assembly, mechanism motion, elastic deformation of the links and joints due to the force and thermal expansion []. There are several literature exists on the effect of manufacturing tolerances on the accuracy of

*Address all correspondence to this author. the parallel manipulators [1]. In [2], a forward and inverse error bound analysis presented to find the error bound in the pose of the end effector for a Stewart platform when the joint error bounds are given and vice versa. The sensitivity analysis for a three degrees-of-freedom translational parallel kinematic machine with orthogonal linear joints is reported in [3]. They have used linkage kinematic analysis and differential vector method to study the influence of the length variation on the pose of the end-effector.

One of the highly addressed problem associated with the end-effector pose error is the manipulator stiffness, which defines the positioning error due to the external loading while executing a specific task by the manipulator. A nonlinear stiffness model for the manipulators with the passive joints is presented in []. Pashkevich et al [4] proposed a novel calibration approach for the Orthoglide based on the observations of the manipulator leg parallelism. A numerical procedure presented in [5], which is used to compute the pose error due to clearances and elastic deformations along a pick-and-place trajectory of the $5 \mathrm{R}$ planar parallel manipulator. In [6], an error prediction model is proposed for overconstrained and nonoverconstrained parallel manipulators and also presents the influence of the joint tolerance on the pose error of the manipulators. A large number of literatures are available on the influence of the statics than the influence of dynamics on computing the error in the pose of the parallel manipulator. A methodology is presented in [8] to project the trajectories in the joint space using Gröbner based elimination methods. This paper mainly focuses on the estimation of error in the pose of end effector due to the joint errors. The proposed error model which is based on the dynamic properties (joint velocities and acceleration) of the Orthoglide helps in estimating the error in the workspace.

The outline of this paper is as follows. We first introduced the direct and inverse kinematic model of the Orthoglide with parallel singularities. In the next section we have defined five different trajectories using quintic polynomial for the Orthoglide 5 -axis, which has been 
developed at IRCCyN. We then proposed the error model based on the joint velocities, acceleration and joint errors which is coming from the Orthoglide. In the later section we have presented the estimation of the error in the pose of the end-effector with Newton and Gröbner based elimination methods.

\section{ARCHITECTURE \& KINEMAT- ICS: ORTHOGLIDE 5-AXIS}

The Orthoglide 5-axis is a hybrid parallel kinematics machine, which consists of a 3 -DOF translational manipulator (Orthoglide 3-axis) and a 2-DOF parallel spherical manipulator (the Agile Eye 2-axis) as two of the main components []. The manipulator under study is a semi industrial prototype of the Orthoglide 5-axis, which is designed and developed at IRCCyN and manufactured by Symetry [], shown in Fig. 1.

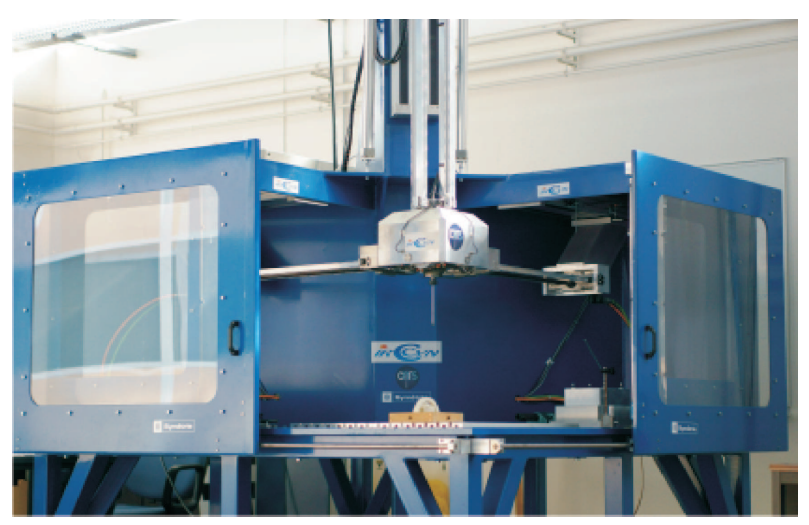

Figure 1: Semi industrial prototype of the Orthoglide 5Axis

Orthoglide 5-axis uses 1103 DSPACE card as a control hardware with a $933 \mathrm{MHz}$ PowerPC. The trajectory planning is done using Matlab and an optical fiber is used to send the data to DSPACE card[]. Two different types of motors are used to actuate the spherical and translational assembly. Two Harmonic Drives FFA-20-80-HE2048 are used to actuate 2-DOF parallel spherical manipulator, which has $3.27 \mathrm{rad} / \mathrm{s}$ as the maximum angular velocity and $270 \mathrm{rad} / \mathrm{s}^{2}$ as the maximum angular acceleration. Orthoglide 3-axis uses three Parvex Brushless NX430EAF coupled with Kinetic TDU 200 ball screws as actuators which has a maximum linear velocity is equal to $1.2 \mathrm{~m} / \mathrm{s}$ and maximum acceleration of $13 \mathrm{~m} / \mathrm{s}^{2}$. The actuator positions are sampled at $9 \mathrm{KHz}$ and actuator velocities are computed using $200 \mathrm{~Hz}$ low pass filter. The gravity effect is reduced using pneumatic compensator which is mounted along the vertical axis.

Equation (1) with $l=310 \mathrm{~mm}$ represents the constraint equations of the Orthoglide 3 -axis, which defines the motion of three orthogonal linear actuators with the actuated variables $\rho=\left[\rho_{1}, \rho_{2}, \rho_{3}\right]$ and the pose variables $\mathbf{X}=[x, y, z]$ are:

$$
\begin{aligned}
F(\rho, \mathbf{X}): & \left(x-\rho_{1}\right)^{2}+y^{2}+z^{2}=l^{2} \\
x^{2}+\left(y-\rho_{2}\right)^{2}+z^{2} & =l^{2} \\
x^{2}+y^{2}+\left(z-\rho_{3}\right)^{2} & =l^{2}
\end{aligned}
$$

$\mathbf{A}_{i} \mathbf{B}_{i}$ is equal to $\rho_{i}$, where $\rho_{i}$ represents the prismatic joint variables whereas $\mathbf{X}$ represents the position vector of the tool center point as it is shown in the Fig. 2. In the following sub sections direct and inverse kinematics model are derived for the Orhoglide 3-axis. Without joint limits there exists two assembly modes and eight working modes for the Orthoglide.

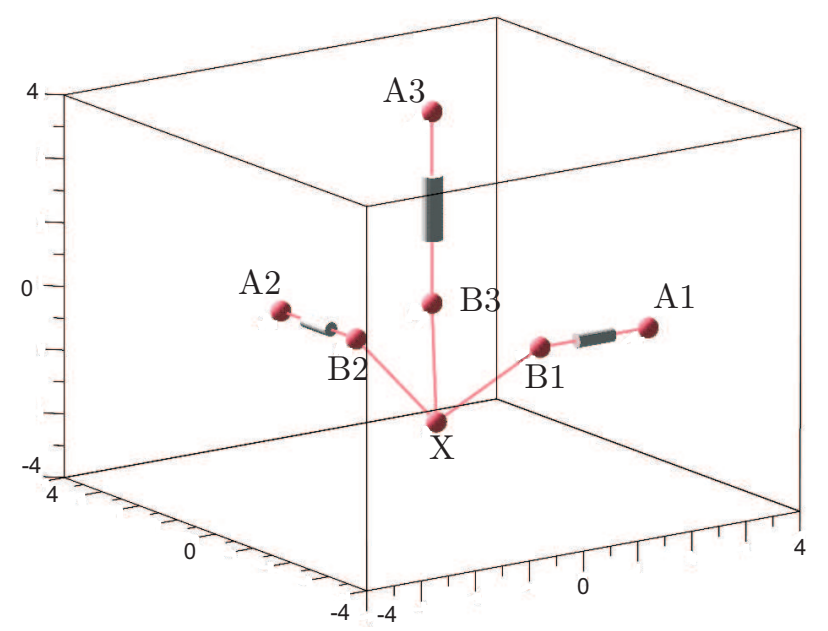

Figure 2: Simplified architecture of the Orthoglide for the simulation and analysis.

\section{Direct Kinematics Model: $\mathbf{X}=\beta(\boldsymbol{\rho})$}

The problem of determining the pose of the end-effector of the manipulator for a given value of joint parameters is termed as a direct kinematic problem (DKP). With the algebraic modelization, there are chances of getting several solutions for DKP i.e the manipulator can be assembled in the different ways for a set of actuator values. The number of solutions for the DKP is referred as the assembly modes of the robot. There exist two real solutions for DKP or two assembly modes for the Orthoglide. Equation (2) represents the direct kinematic model for the Orthoglide 3-axis.

$$
\begin{aligned}
& \beta(\boldsymbol{\rho}): x=\frac{1}{2 \rho_{1}} \frac{\rho_{1}^{4} \rho_{2}^{2}+\rho_{1}^{4} \rho_{3}^{2}-\sqrt{\mathbf{N}} \rho_{3}}{\mathbf{M}} \\
& y=\frac{1}{2 \rho_{2}} \frac{\rho_{1}^{4} \rho_{2}^{2}+\rho_{2}^{4} \rho_{3}^{2}-\sqrt{\mathbf{N}} \rho_{3}}{\mathbf{M}} \\
& z=\frac{1}{2 \rho_{3}} \frac{\rho_{1}^{4} \rho_{3}^{2}+\rho_{2}^{4} \rho_{3}^{2}-\sqrt{\mathbf{N}} \rho_{3}}{\mathbf{M}} \\
& \text { where } \mathbf{M}=\rho_{1}^{2} \rho_{2}^{2}+\rho_{1}^{2} \rho_{3}^{2}+\rho_{2}^{2} \rho_{3}^{2} \\
& \mathbf{N}=\quad-\rho_{\mathbf{1}}^{\mathbf{6}} \rho_{\mathbf{2}}^{\mathbf{4}}-\rho_{\mathbf{1}}^{\mathbf{6}} \rho_{\mathbf{2}}^{\mathbf{2}} \rho_{\mathbf{3}}^{\mathbf{2}}-\rho_{\mathbf{1}}^{\mathbf{4}} \rho_{\mathbf{2}}^{\mathbf{6}}-\mathbf{2}_{\mathbf{1}}^{\mathbf{4}} \rho_{\mathbf{2}}^{\mathbf{4}} \rho_{\mathbf{3}}^{\mathbf{2}}-\rho_{\mathbf{1}}^{\mathbf{4}} \rho_{\mathbf{2}}^{\mathbf{2}} \rho_{\mathbf{3}}^{\mathbf{4}}- \\
& \rho_{1}^{2} \rho_{2}^{6} \rho_{3}^{2}-\rho_{1}^{2} \rho_{2}^{4} \rho_{3}^{4}+384400\left(\rho_{1}^{4} \rho_{2}^{4}+\rho_{1}^{4} \rho_{2}^{2} \rho_{3}^{2}+\rho_{1}^{2} \rho_{2}^{4} \rho_{3}^{2}\right)
\end{aligned}
$$


Solving Eq. (1) for $\mathbf{X}$ gives two solutions corresponding to the two assembly modes of the Orthoglide. Equation (2) represents only one solution which corresponds to the required assembly modes. $\beta(\boldsymbol{\rho})$, the direct kinematic model is used to compute the projection of the joint errors in the workspace of the Orthoglide [9].

\section{Inverse Kinematics Model: $\rho=\gamma(\mathbf{X})$}

Similarly the problem of determining the joint actuators value for a given pose of a end-effector is termed as a inverse kinematic problem (IKP). The number of solutions for the IKP is referred as the working modes of the robot. There exist eight real solutions for IKP or the working modes for the Orthoglide 3 -axis that can be computed using the Eq. (3).

$$
\begin{aligned}
\gamma(\mathbf{X}): \rho_{1} & =x \pm \sqrt{-y^{2}-z^{2}+96100} \\
\rho_{2} & =y \pm \sqrt{-x^{2}-z^{2}+96100} \\
\rho_{3} & =z \pm \sqrt{-x^{2}-y^{2}+96100}
\end{aligned}
$$

\section{Parallel Singularities}

Singularities plays an important role while defining the trajectory for a specific task. The error in the poses of the end-effector gradually increases when it approaches singular configuration. Differentiating Eq. (1) with respect to time leads to the velocity model:

$$
\mathbf{A} \dot{\mathbf{X}}+\mathbf{B} \dot{\rho}=\mathbf{0} \quad \text { where } \quad \mathbf{A}=\frac{\partial \mathbf{F}}{\partial \mathbf{X}}, \quad \mathbf{B}=\frac{\partial \mathbf{F}}{\partial \boldsymbol{\rho}}
$$

The matrices $\mathbf{A}$ and $\mathbf{B}$ are respectively the directkinematics and the inverse-kinematics Jacobian matrices of the manipulator. These matrices are used for characterizing different kinds of singularities. The parallel singularities occur whenever $\operatorname{det}(\mathbf{A})=0$, i.e, the mapping from tool velocity space to the joint velocity space is ill-conditioned and the serial singularities occur whenever $\operatorname{det}(\mathbf{B})=0$. The parallel singular configurations are located inside the workspace. They are particularly undesirable because the manipulator cannot resist to any forces and its control is lost. Parallel singularities occurs when the target point and the center of prismatic joints are lie in the same plane or when all three links parallel to each other.

$$
\begin{array}{r}
\operatorname{det}(\mathbf{A})=-8 \rho_{1} \rho_{2} \rho_{3}+8 \rho_{1} \rho_{2} z+8 \rho_{1} \rho_{3} y+8 \rho_{2} \rho_{3} x \\
\operatorname{det}(\mathbf{A}) \mapsto \varepsilon(\rho) \quad \operatorname{det}(\mathbf{A}) \mapsto \xi(\mathbf{X}) \\
\varepsilon(\rho)=\rho_{1}^{4} \rho_{2}^{2}+\rho_{1}^{4} \rho_{3}^{2}+\rho_{1}^{2} \rho_{2}^{4}+2 \rho_{1}^{2} \rho_{2}^{2} \rho_{3}^{2}+\rho_{1}^{2} \rho_{3}^{4}+ \\
\rho_{2}^{4} \rho_{3}^{2}+\rho_{2}^{2} \rho_{3}^{4}-16 \rho_{1}^{2} \rho_{2}^{2}-16 \rho_{1}^{2} \rho_{3}^{2}-16 \rho_{2}^{2} \rho_{3}^{2}
\end{array}
$$

In Eq. (5), $\operatorname{det}(\mathbf{A})$ is the parallel singularity of the Orthoglide and $\xi(\mathbf{X})$ is the projection of $\operatorname{det}(\mathbf{A})$ in the cartesian space. $\varepsilon(\rho)$ is the projection of parallel singularity $\operatorname{det}(\mathbf{A})$ in the joint space of the Orthoglide. The mathematical expression for $\xi(\mathbf{X})$ is not mentioned in Eq. (5) due to the lack of space. Figure 3 shows the projection
$\xi(\mathbf{X})$ in the workspace of the Orthoglide. The Gröbner basis and cylindrical algebraic decomposition (CAD) algorithms are used to compute and plot the workspace and parallel singularities of the Orthoglide []. The degree of this characteristic surface is 18 and it represents the singularities associated with the eight working modes.

\section{TRAJECTORY PLANNING}

The most simple and suitable technique for planning the trajectory for a specific task is always to define the path in the workspace $\mathbf{X}=[x, y, z]$ and control loop in the joint space $\rho=\left[\rho_{1}, \rho_{2}, \rho_{3}\right]$ of the manipulator. Equation (6) represents the torque control structure for the actuators motion []. As it can be observed from Eq. (6), the torque $\Gamma_{i}$ also depends on the dynamics of the manipulator which further enhances the classical PID control scheme.

$$
\begin{gathered}
\Gamma_{i}=M\left(\ddot{\rho}_{i}+\mathbf{K}_{\mathbf{P}}\left(\rho_{\mathbf{i}}^{\mathbf{d}}-\rho_{\mathbf{i}}\right)+\mathbf{K}_{\mathbf{I}} \int_{\mathbf{t}_{\mathbf{o}}}^{\mathbf{t}}\left(\rho_{\mathbf{i}}^{\mathbf{d}}-\rho_{\mathbf{i}}\right)+\mathbf{K}_{\mathbf{D}}\left(\rho_{\mathbf{i}}^{\mathbf{d}}-\rho_{\mathbf{i}}\right)\right) \\
\text { where } \quad i=1,2,3 \quad \text { and } \quad M=91.6278 \mathrm{Kg}
\end{gathered}
$$

For the study five similar circular trajectories $\phi_{1}(t)$, $\phi_{2}(t), \phi_{3}(t), \phi_{4}(t)$ and $\phi_{5}(t)$ are defined but with the different center locations $c_{1}, c_{2}, c_{3}, c_{4}$ and $c_{5}$, respectively, inside the Orthoglide's workspace. These trajectories can be seen in Fig. 3 which are labeled as 1,2..5 with workspace and parallel singularity surfaces. The trajectories $\phi_{i}(t)$ : $\left[\tau_{x_{i}}, \tau_{y_{i}}, \tau_{z_{i}}\right]$ in Eq. (8) are defined in parametric form in the Cartesian space using fifth order polynomial equation with different center location which is shown in Eq. (7).

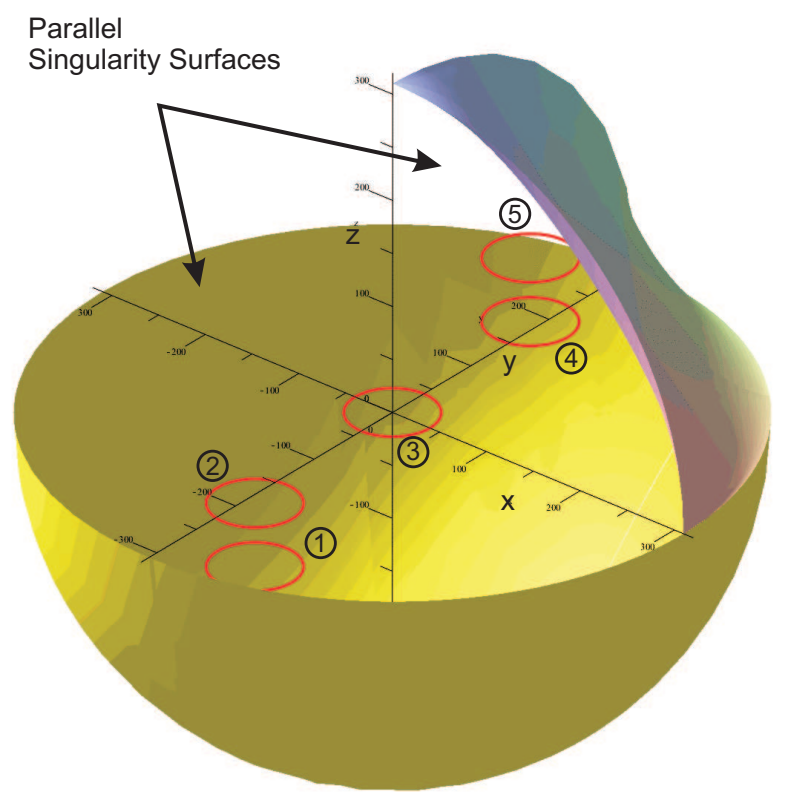

Figure 3: Location of the trajectories $\phi_{i}(t)$ along with the workspace and parallel singularity surface $\xi(\mathbf{X})$. CAD algorithm is used to plot these surfaces.

The execution time is set to one seconds for all the trajectories. The trajectories for Orthoglide 5-axis are defined 


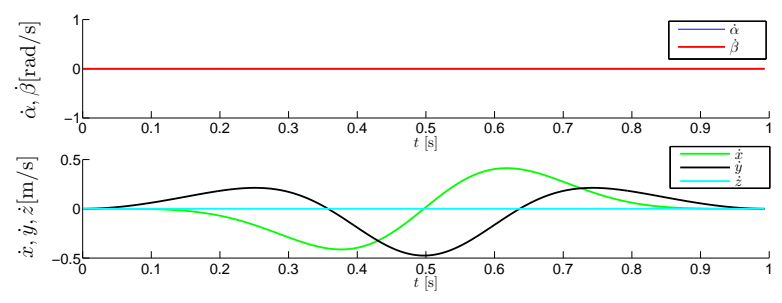

Figure 4: Cartesian Velocities along the trajectories $\phi_{i}(t)$

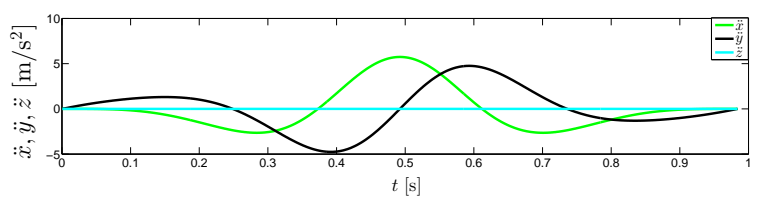

Figure 5: Cartesian Acceleration along the trajectories $\phi_{i}(t)$

such that $\alpha$ and $\beta$ are equal to zero i.e, there exists only translational motion along $x, y$ and $z$ direction.

$$
\begin{aligned}
& c_{1}:\left[c_{x_{1}}, c_{y_{1}}, c_{z_{1}}\right]: \rightarrow[-80,-80,-140] \\
& c_{2}:\left[c_{x_{2}}, c_{y_{2}}, c_{z_{2}}\right]: \rightarrow[-80,-80,-80] \\
& c_{3}:\left[c_{x_{3}}, c_{y_{3}}, c_{z_{3}}\right]: \rightarrow[00,00,00] \\
& c_{4}:\left[c_{x_{4}}, c_{y_{4}}, c_{z_{4}}\right]: \rightarrow[80,80,80] \\
& c_{5}:\left[c_{x_{5}}, c_{y_{5}}, c_{z_{5}}\right]: \rightarrow[80,80,140]
\end{aligned}
$$

Differentiating Eq. (8) with respect to time gives us Cartesian velocities $\tau_{x_{i}}^{\cdot}, \tau_{y_{i}}^{\cdot}$ and $\tau_{z_{i}}^{\cdot}$ along $x, y$ and $z$ direction respectively, which is shown in Eq. (9). Similarly, Cartesian accelerations can be obtained by differentiating Cartesian velocities i.e, Eq. (9) with respect to time.

$$
\begin{array}{r}
\phi_{i}(t): \tau_{x_{i}}=c_{x_{i}}+40 \sin \left(2 t^{3}\left(6 t^{2}-15 t+10\right) \pi\right)-x \\
\tau_{y_{i}}=c_{y_{i}}+40 \cos \left(2 t^{3}\left(6 t^{2}-15 t+10\right) \pi\right)-y \\
\tau_{z_{i}}=c_{z_{i}}-z \quad \forall t \in[0,1]
\end{array}
$$

Figure 4 and 5 shows the plot of Cartesian velocities and accelerations, respectively, along the trajectory $\phi_{i}(t)$. All the trajectories has the same Cartesian velocities and accelerations.

$\phi_{1}(t), \phi_{2}(t), \phi_{3}(t), \phi_{4}(t)$ and $\phi_{5}(t)$ are the trajectories which are defined in the workspace. To project these trajectories in the joint space, it is necessary to formulate a system of equations corresponding to each trajectory which also consists the kinematic equations of the manipulator.

$$
\begin{array}{rr}
\tau_{x_{i}}= & 2400 t^{2} \pi\left(t^{2}-2 t+1\right) \cos \left(2 t^{3}\left(6 t^{2}-15 t+10\right) \pi\right) \\
\tau_{y_{i}}=-2400 t^{2} \pi\left(t^{2}-2 t+1\right) \sin \left(2 t^{3}\left(6 t^{2}-15 t+10\right) \pi\right) \\
\dot{\tau_{z_{i}}}=0 \quad \forall t \in[0,1]
\end{array}
$$

$\Psi_{1}, \Psi_{2}, \Psi_{3}, \Psi_{4}$ and $\Psi_{5}$ are the corresponding systems of equations for the Trajectories $1,2,3,4$, and 5, respectively (see Eq. (10)).

$$
\begin{aligned}
& \Psi_{1}(\mathbf{X}, \rho, \mathbf{t})=\left[\left[\tau_{x_{1}}, \tau_{y_{1}}, \tau_{z_{1}}\right], F(\rho, \mathbf{X})\right] \\
& \Psi_{2}(\mathbf{X}, \rho, \mathbf{t})=\left[\left[\tau_{x_{2}}, \tau_{y_{2}}, \tau_{z_{2}}\right], F(\rho, \mathbf{X})\right] \\
& \Psi_{3}(\mathbf{X}, \rho, \mathbf{t})=\left[\left[\tau_{x_{3}}, \tau_{y_{3}}, \tau_{z_{3}}\right], F(\rho, \mathbf{X})\right] \\
& \Psi_{4}(\mathbf{X}, \rho, \mathbf{t})=\left[\left[\tau_{x_{4}}, \tau_{y_{4}}, \tau_{z_{4}}\right], F(\rho, \mathbf{X})\right] \\
& \Psi_{5}(\mathbf{X}, \rho, \mathbf{t})=\left[\left[\tau_{x_{5}}, \tau_{y_{5}}, \tau_{z_{5}}\right], F(\rho, \mathbf{X})\right]
\end{aligned}
$$

Each system of equation is projected in the joint space as $\Upsilon_{1}(\rho, \mathbf{t}), \Upsilon_{2}(\rho, \mathbf{t})$ and $\Upsilon_{3}(\rho, \mathbf{t})$ (see Eq. (11)). By solving $\Upsilon_{1}(\rho), \Upsilon_{2}(\rho)$ and $\Upsilon_{3}(\rho)$ for $\rho$, we get the corresponding parametric equations for the trajectories in the joint space. There are two different ways to compute these projections. The first method uses Gröbner based elimination algorithms [] and the second method is to directly substitute the $\left[\tau_{x_{1}}, \tau_{y_{1}}, \tau_{z_{1}}\right]$ in inverse kinematic model (Equation (3)).

$$
\begin{array}{lll}
\Psi_{1}(\mathbf{X}, \rho, \mathbf{t}) & \mapsto \Upsilon_{1}(\rho, \mathbf{t}) & \forall t \in[0,1] \\
\Psi_{2}(\mathbf{X}, \rho, \mathbf{t}) \mapsto \Upsilon_{2}(\rho, \mathbf{t}) & \forall t \in[0,1] \\
\Psi_{3}(\mathbf{X}, \rho, \mathbf{t}) \mapsto \Upsilon_{3}(\rho, \mathbf{t}) & \forall t \in[0,1] \\
\Psi_{4}(\mathbf{X}, \rho, \mathbf{t}) \mapsto \Upsilon_{4}(\rho, \mathbf{t}) & \forall t \in[0,1] \\
\Psi_{5}(\mathbf{X}, \rho, \mathbf{t}) \mapsto \Upsilon_{5}(\rho, \mathbf{t}) & \forall t \in[0,1]
\end{array}
$$

Solving $\Upsilon_{1}(\rho, \mathbf{t})$ and $\Upsilon_{2}(\rho, \mathbf{t})$ for $\rho$ gives eight possible solutions for trajectories $\phi_{i}(t)$, but only one feasible solution exists which lies inside the joint space, which corresponds to each trajectories. The first coloum in the Figure, i.e, Figure $\left(a_{i}\right)$ represents the joint actuators value along the trajectory $\Psi_{1}$, wheras second and third coloumn, i.e, Figure $\left(b_{i}\right) \&\left(c_{i}\right)$ represents the joint velocities and accelerations, respectively.

\section{ERROR ANALYSIS}

During the execution of any given trajectory by the manipulator, there always exist some differences between the desired actuator values and the actual actuator values at any given instance of time. These differences are recorded by sensors which are generally attached with the actuators. Figure shows the recorded values of joint errors while executing the trajectories by the Orthoglide 5-axis for the translational part.

The different parameters associated with the recorded joint errors $\Delta \rho_{i}$ along the the trajectories $\phi_{i}(t)$ in Fig. such as maximum, minimum and mean absolute values are presented in Table 1 . The maximum error in $\rho_{1}$ is more $[697 \mu \mathrm{m}]$ for the trajectory $T_{5}$, whereas less $[584.8 \mu \mathrm{m}]$ for the trajectory $T_{1}$. The mean absolute error in $\rho_{1}$ is the maximum $[171.2 \mu \mathrm{m}]$ for the trajectory $T_{5}$ and minimum $[125.8 \mu \mathrm{m}]$ for the trajectory $T_{1}$. The maximum error in $\rho_{2}$ is more $[784 \mu \mathrm{m}]$ for the trajectory $T_{1}$, whereas less [565 $\mu m]$ for the trajectory $T_{5}$. The mean absolute error in $\rho_{2}$ is the maximum $[220.2 \mu \mathrm{m}]$ for the trajectory $T_{2}$ and minimum $[135.8 \mu \mathrm{m}]$ for the trajectory $T_{4}$. The maximum error in $\rho_{3}$ is more $[558 \mu \mathrm{m}]$ for the trajectory $T_{1}$, whereas less $[020.2 \mu \mathrm{m}]$ for the trajectory $T_{1}$. The mean absolute error in $\rho_{3}$ is the maximum $[167.7 \mu \mathrm{m}]$ for the trajectory $T_{5}$ and minimum $[011.5 \mu \mathrm{m}]$ for the trajectory $T_{3}$. 


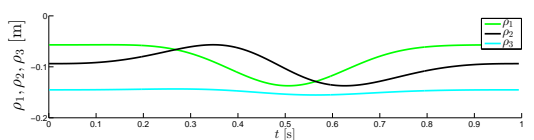

$\left(a_{1}\right)$

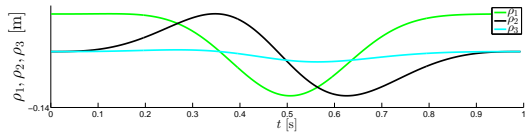

$\left(a_{2}\right)$

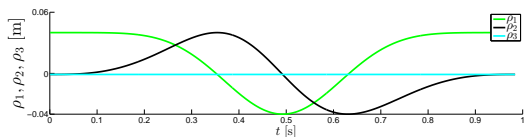

$\left(a_{3}\right)$

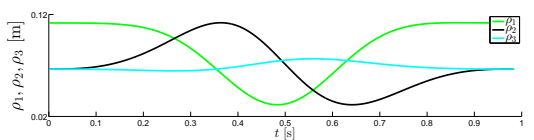

$\left(a_{4}\right)$

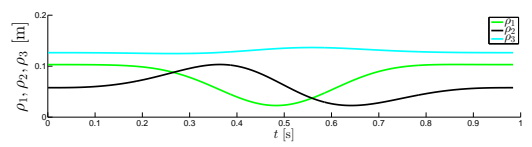

$\left(a_{5}\right)$

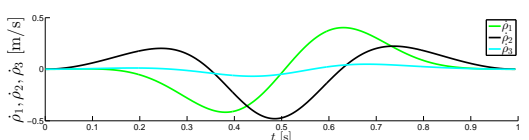

$\left(b_{1}\right)$

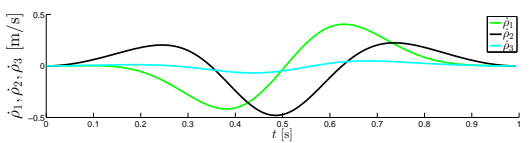

$\left(b_{2}\right)$

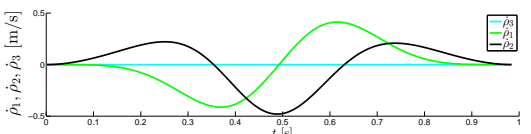

$\left(b_{3}\right)$

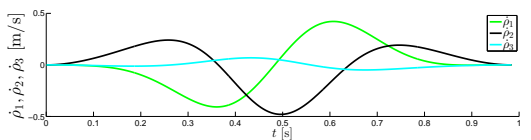

$\left(b_{4}\right)$

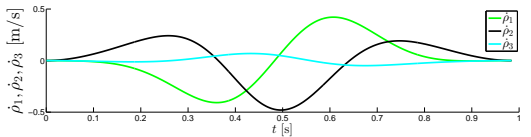

$\left(b_{5}\right)$

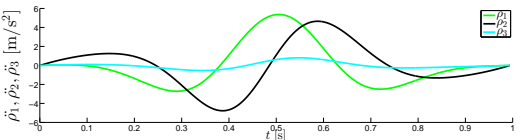

$\left(c_{1}\right)$

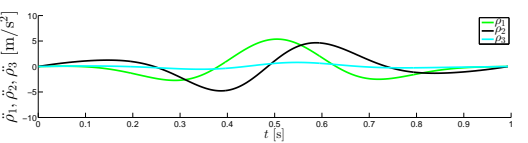

$\left(c_{2}\right)$

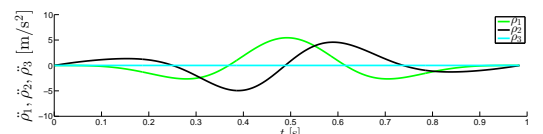

(c $\left.c_{3}\right)$

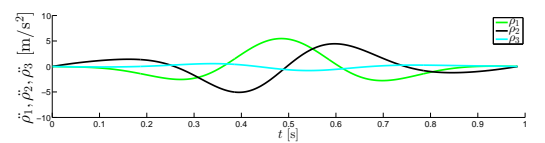

(c $\left.c_{4}\right)$

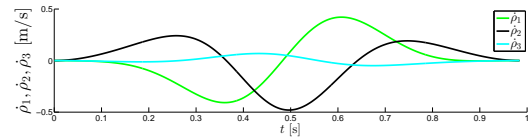

$\left(c_{5}\right)$

Figure 6: Joint parameters value along trajectory $\phi_{1}(\mathrm{t}):[-80,-80,-140], \phi_{2}(\mathrm{t}):[-80,-80,-80], \phi_{3}(\mathrm{t}):[00,00,00]$, $\phi_{4}(\mathrm{t}):[80,80,140], \phi_{5}(\mathrm{t}):[80,80,140]$ Joint positions $\left(a_{i}\right)$ Joint velocities $\left(b_{i}\right)$ Joint accelerations $\left(c_{i}\right)$, where $i=$ $1,2,3,4,5$ which represents different trajectories

Table 1: The Minimum, Maximum and mean absolute values of joint errors along the trajectories

\begin{tabular}{|c|c|c|c|c|c|c|c|c|c|}
\hline \multirow{2}{*}{$\frac{\text { Errors }(\mu m) \rightarrow}{\text { Trajectories } \downarrow}$} & \multicolumn{3}{|c|}{$\Delta \rho_{1}$} & \multicolumn{3}{|c|}{$\Delta \rho_{2}$} & \multicolumn{3}{|c|}{$\Delta \rho_{3}$} \\
\hline & Min & $\operatorname{Max}$ & Avg & Min & Max & Avg & Min & $\operatorname{Max}$ & Avg \\
\hline$T_{1}:[-80,-80,-140]$ & -280.6 & 584.8 & 125.8 & -639.1 & 784.0 & 212.1 & -401.4 & 558.0 & 151.0 \\
\hline$T_{2}:\left[\begin{array}{ll}-80,-80, & -80]\end{array}\right.$ & -312.7 & 626.6 & 130.6 & -665.2 & 777.7 & 220.2 & -424.5 & 529.3 & 159.5 \\
\hline$T_{3}:\left[\begin{array}{lll}00, & 00, & 00]\end{array}\right.$ & -272.4 & 663.9 & 149.0 & -615.1 & 666.1 & 204.2 & -020.2 & 020.2 & 011.5 \\
\hline $80,80,80]$ & -312.6 & 685.9 & 161.2 & -636.7 & 566.6 & 135.8 & -572.9 & 410.1 & 167.0 \\
\hline $80,80,140]$ & -314.5 & 697.0 & 171.2 & -630.9 & 565.0 & 138.6 & -565.5 & 427.2 & 167.7 \\
\hline
\end{tabular}


There may exist several factors which affect these joints errors. It has also been observed that with the change of starting pose, accuracy of the manipulator also changes for a specified task. By comparing the data for different trajectories From Table 1, it can be inferred that by changing the location of the trajectories inside the workspace of the Orthoglide, there is a significant change in the joint errors. It is difficult to choose the best suitable location for a trajectory inside the workspace based on the joint errors because we have to evaluate their images in the workspace.

\section{ERROR MODEL}

One of the essential steps in computing the projection of the joint errors in the workspace is to model the joint errors. The proposed error model is based on the experimental data and the dynamics, i.e, joint velocities and accelerations of the Orthoglide 5-axis.

$$
\Delta \rho_{i}^{m}=k_{1}+k_{2} \dot{\rho}_{i}+k_{3} \ddot{\rho}_{i}
$$

The Equation (12) represents the proposed error model in which $k_{1}, k_{2}$ and $k_{3}$ are the constants and $\dot{\rho}_{i}$ and $\ddot{\rho}_{i}$ are the joint velocity and acceleration, respectively. The modeled error $\Delta \rho_{i}{ }^{m}$ and the experimental data $\Delta \rho_{i}$ from the manipulator for joint errors are compared to get the best suited values for $k_{1}, k_{2}$ and $k_{3}$ for the best fit.

$$
k_{1}=\frac{4}{100000} \quad k_{2}=\frac{5}{10000000} \quad k_{3}=\frac{24}{100000000}
$$

The computed values $k_{1}, k_{2}$ and $k_{3}$ is shown in Eq. (12), by substituting these values in Eq. (12), we will get Eq. (14), which represents the proposed error model for

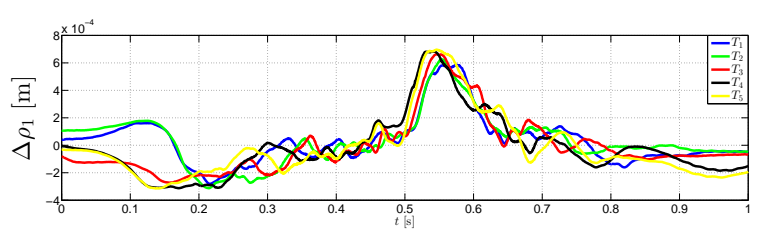

(a)

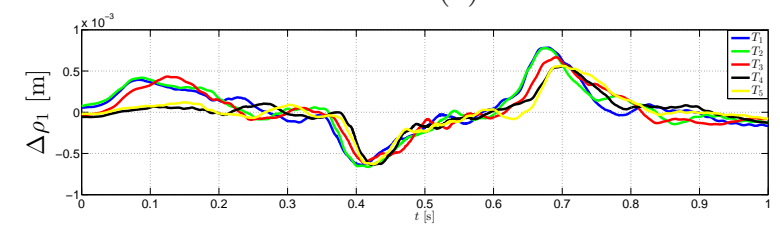

(b)

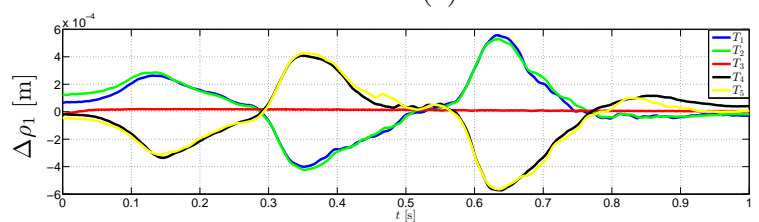

(c)

Figure 7: Joint Errors in $\Delta \rho_{1}$ (a) $\Delta \rho_{2}$ (b) and $\Delta \rho_{3}$ (c) along the trajectories $\phi_{i}(t)$, Experimental data from the Orthoglide 5-axis the Orthoglide. More optimized algorithm can be used to compute the more appropriate values for the constants.

$$
\begin{aligned}
& \Delta \rho_{1}{ }^{m}=\frac{4}{100000}+\frac{5}{10000000} \dot{\rho_{1}}+\frac{24}{100000000} \ddot{\rho}_{1} \\
& \Delta \rho_{2}{ }^{m}=\frac{4}{100000}+\frac{5}{10000000} \dot{\rho}_{2}+\frac{24}{100000000} \ddot{\rho}_{2} \\
& \Delta \rho_{3}{ }^{m}=\frac{4}{100000}+\frac{5}{10000000} \dot{\rho}_{3}+\frac{24}{100000000} \ddot{\rho}_{3}
\end{aligned}
$$

The black dotted line in the Fig. represents the joint errors which are obtained from the Orthogolide while executing the trajectory. The red line in the Fig. is the modeled error, which further will be used to compute the image of the joint errors in the workspace of the manipulator.

\section{PROJECTION OF JOINT ER- RORS IN THE WORKSPACE}

Newton method is used to compute the image of joint errors in the workspace of the manipulator. By differentiating Eq. (3), i.e, inverse kinematic equations and substituting the values of proposed error model $\Delta \rho_{i}{ }^{m}$, i.e $e_{1}=\Delta \rho_{1}{ }^{m}, e_{2}=\Delta \rho_{2}{ }^{m}$ and $e_{3}=\Delta \rho_{3}{ }^{m}$ in Eq. (15), we will get the image of joint errors $\Delta x, \Delta y$ and $\Delta z$ in the workspace of the manipulator.

$$
\begin{aligned}
\Delta x^{2} & =\left(\frac{\partial x}{\partial \rho_{1}}\right)^{2} e_{1}^{2}+\left(\frac{\partial x}{\partial \rho_{2}}\right)^{2} e_{2}^{2}+\left(\frac{\partial x}{\partial \rho_{3}}\right)^{2} e_{3}^{2} \\
\Delta y^{2} & =\left(\frac{\partial y}{\partial \rho_{1}}\right)^{2} e_{1}^{2}+\left(\frac{\partial y}{\partial \rho_{2}}\right)^{2} e_{2}^{2}+\left(\frac{\partial y}{\partial \rho_{3}}\right)^{2} e_{3}^{2} \\
\Delta z^{2} & =\left(\frac{\partial z}{\partial \rho_{1}}\right)^{2} e_{1}^{2}+\left(\frac{\partial z}{\partial \rho_{2}}\right)^{2} e_{2}^{2}+\left(\frac{\partial z}{\partial \rho_{3}}\right)^{2} e_{3}^{2}
\end{aligned}
$$

The different parameters associated with the projected joint errors in the workspace $\Delta x, \Delta y$ and $\Delta z$ along the the trajectories $\phi_{i}(t)$ in Fig. are presented in Table 2. The maximum error in $\mathbf{x}$ is more $[3.0 \mathrm{~mm}]$ for the trajectory $T_{5}$, whereas less $[1.30 \mathrm{~mm}]$ for the trajectory $T_{3}$. The mean absolute error in $\mathbf{x}$ is the maximum $[1.20 \mathrm{~mm}]$ for the trajectory $T_{5}$ and minimum $[0.40 \mathrm{~mm}]$ for the trajectory $T_{3}$. The maximum error in $y$ is more $[3.20 \mathrm{~mm}]$ for the trajectory $T_{5}$, whereas less $[565 \mathrm{~mm}]$ for the trajectory $T_{3}$. The mean absolute error in $\mathbf{y}$ is the maximum $[3.20 \mathrm{~mm}]$ for the trajectory $T_{2}$ and minimum $[1.20 \mathrm{~mm}]$ for the trajectory $T_{3}$. The maximum error in $\mathbf{z}$ is more $[2.50 \mathrm{~mm}]$ for the trajectory $T_{5}$, whereas less $[0.20 \mathrm{~mm}]$ for the trajectory $T_{3}$. The mean absolute error in $\mathbf{z}$ is the maximum $[0.88 \mathrm{~mm}]$ for the trajectory $T_{5}$ and minimum $[0.08 \mathrm{~mm}]$ for the trajectory $T_{3}$.

The mean absolute error in $\mathbf{y}$ is the maximum [3.20 $\mathrm{mm}$ ] for the trajectory $T_{2}$ and minimum $[1.20 \mathrm{~mm}]$ for the trajectory $T_{3}$. The maximum error in $\mathbf{z}$ is more $[2.50 \mathrm{~mm}]$ for the trajectory $T_{5}$, whereas less $[0.20 \mathrm{~mm}]$ for the trajectory $T_{3}$. The mean absolute error in $\mathbf{z}$ is the maximum $[0.88 \mathrm{~mm}]$ for the trajectory $T_{5}$ and minimum $[0.08 \mathrm{~mm}]$ for the trajectory $T_{3}$. The mean absolute error in $\mathbf{y}$ is the 


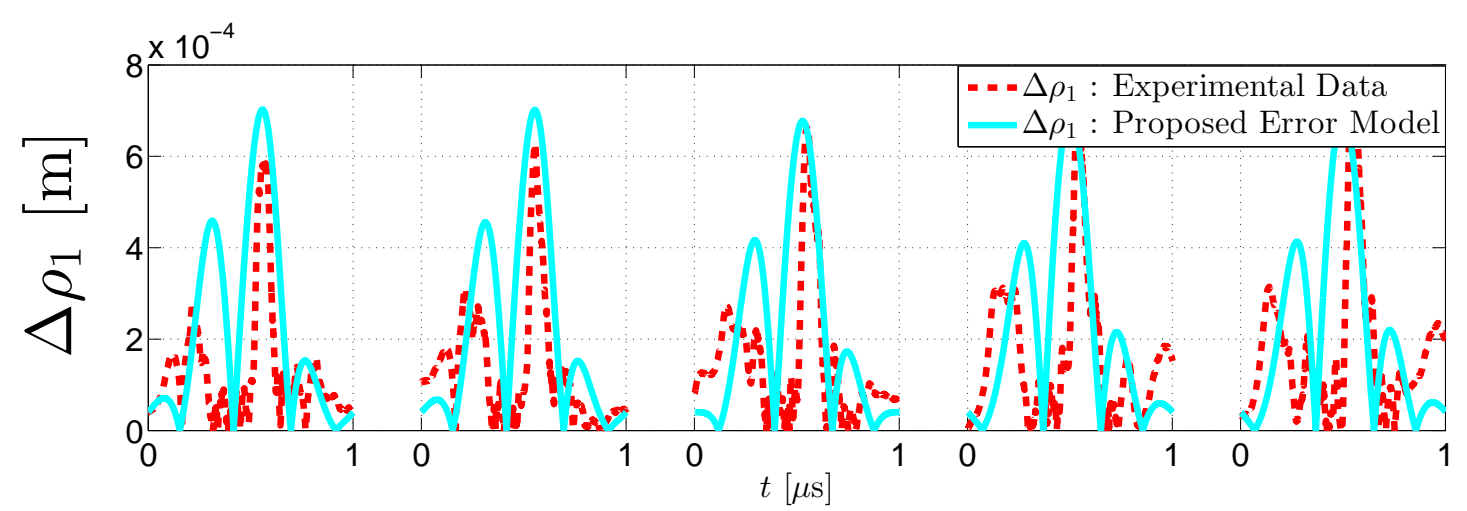

(a)

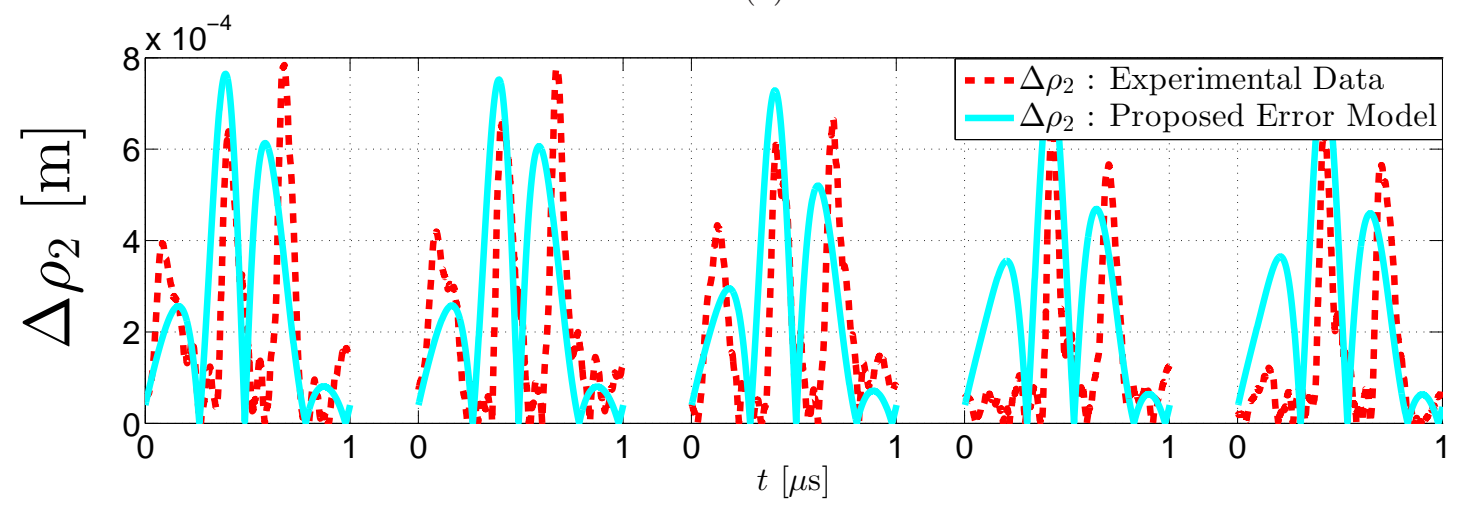

(b)

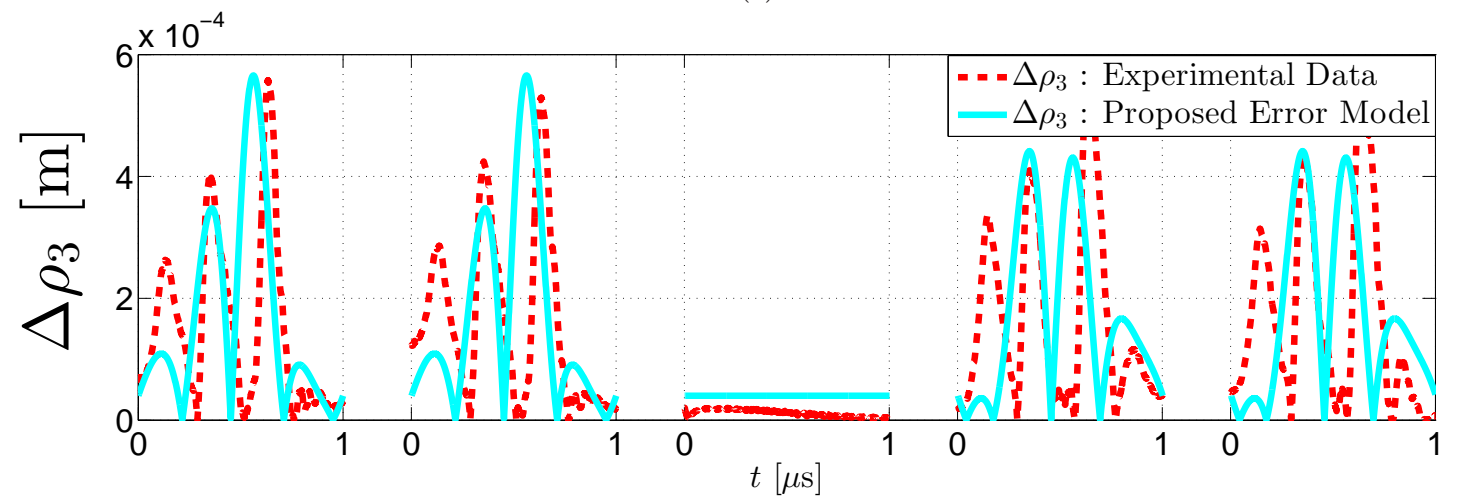

(c)

Figure 8: Joint error $\Delta \rho_{i}$ and proposed error model $\Delta \rho_{i}{ }^{m}$ value along trajectory $\phi_{1}(\mathrm{t})$ : $[-80,-80,-140], \phi_{2}(\mathrm{t})$ : $[-80,-80,-80], \phi_{3}(\mathrm{t}):[00,00,00], \phi_{4}(\mathrm{t}):[80,80,140], \phi_{5}(\mathrm{t}):[80,80,140] \Delta \rho_{1}\left(a_{i}\right) \Delta \rho_{2}\left(b_{i}\right) \Delta \rho_{3}\left(c_{i}\right)$, where $i=$ $1,2,3,4,5$ which represents different trajectories

Table 2: Maximum and mean absolute values of Cartesian position errors

\begin{tabular}{|c|c|c|c|c|c|c|c|}
\hline \multicolumn{2}{|r|}{ Errors $\rightarrow$} & \multicolumn{2}{|c|}{$x$} & & \multicolumn{2}{|c|}{$z$} \\
\hline & ajectories $\downarrow$ & Max & Avg & $\operatorname{Max}$ & Avg & $\operatorname{Max}$ & Avg \\
\hline$T_{1}:[$ & $-80,-80,-140]$ & 1.70 & 0.60 & 1.80 & 0.50 & 1.10 & 0.30 \\
\hline$T_{2}:[-$ & $80,-80, \quad-80]$ & 1.50 & 0.50 & 1.60 & 0.50 & 0.90 & 0.26 \\
\hline$T_{3}:$ & $\left.\begin{array}{lll}00, & 00, & 00\end{array}\right]$ & 1.30 & 0.40 & 1.20 & 0.40 & 0.20 & 0.08 \\
\hline$T_{4}:$ & $80,80, \quad 80]$ & 1.80 & 0.70 & 1.50 & 0.70 & 1.20 & 0.47 \\
\hline$T_{5}$ & $80, \quad 80,140]$ & 3.00 & 1.20 & 3.20 & 1.20 & 2.50 & 0.88 \\
\hline
\end{tabular}




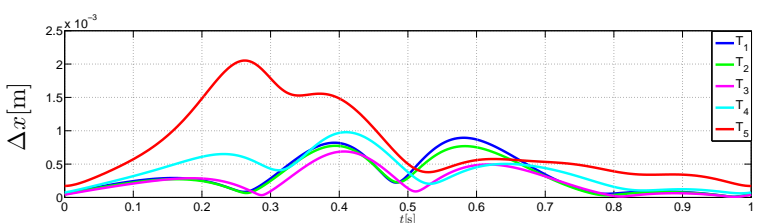

(a)

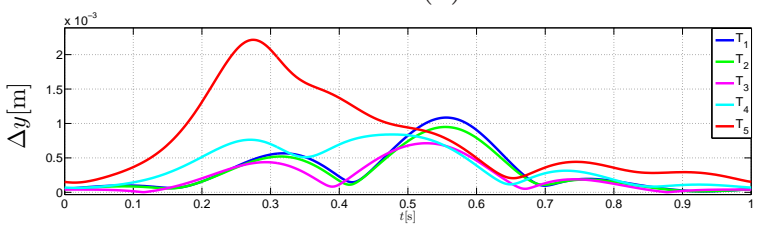

(b)

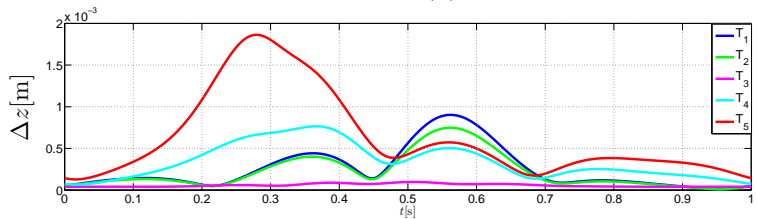

$(c)$

Figure 9: Cartesian Errors in $\mathbf{x}$ (a) $\mathbf{y}$ (b) and $\mathbf{z}$ (c) along the trajectories $\phi_{i}(t)$, Image of the joint errors in the workspace of the manipulator

maximum $[3.20 \mathrm{~mm}]$ for the trajectory $T_{2}$ and minimum $[1.20 \mathrm{~mm}]$ for the trajectory $T_{3}$. The maximum error in $\mathbf{z}$ is more $[2.50 \mathrm{~mm}]$ for the trajectory $T_{5}$, whereas less $[0.20 \mathrm{~mm}]$ for the trajectory $T_{3}$. The mean absolute error in $\mathbf{z}$ is the maximum $[0.88 \mathrm{~mm}]$ for the trajectory $T_{5}$ and minimum $[0.08 \mathrm{~mm}]$ for the trajectory $T_{3}$.

\section{CONCLUSIONS}

This paper dealt with the error modeling of the Orthoglide 5 -axis thanks to experimental data associated with the trajectory planning parameters. The accuracy of the path in the Cartesian workspace was studied for five circle trajectories with the same velocity and acceleration profiles. We have found out that the trajectory closest to the singularity surface admitted the maximum error and the trajectory closest to the isotropic posture the minimum error. These results did not integrate the influence of the starting point and the location of the maximum acceleration along the path but similar results can be found out. Experiments are ongoing to refine the accuracy model by changing the maximum velocity and acceleration.

\section{Acknowledgment}

The work presented in this paper was partially funded by the Erasmus Mundus project "India4EU II".

\section{References}

[1] Wang J. and Masory O., On the accuracy of a Stewart platform. I. The effect of manufacturing tolerances, in Robotics and Automation, Proceedings., 1993 IEEE
International Conference on , vol. 1, pp. 114-120, 1993.

[2] Kim H. S., and Choi Y. J., The kinematic error bound analysis of the Stewart platform, Journal of Robotic Systems, Vol. 17(01), pp. 63-73, 2000.

[3] Caro S., Wenger P., Bennis F., Chablat D., Sensitivity Analysis of the Orthoglide: A Three-DOF Translational Parallel Kinematic Machine, ASME. J. Mech. Des., Vol. 128(2), pp. 392-402, 2005.

[4] Pashkevich A., Chablat D., Wenger P., Kinematics and workspace analysis of a three-axis parallel manipulator the Orthoglide, Robotica, Vol. 24(01), pp. 39-49, 2006.

[5] Aginaga J., Altuzarra O., Macho E., Iriarte X., Assessing Position Error Due to Clearances and Deformations of Links in Parallel Manipulators, ASME. J. Mech. Des., vol. 135(04), pp. 041006, 2013.

[6] Meng J., Zhang D., Li Z., Accuracy Analysis of Parallel Manipulators With Joint Clearance, ASME. J. Mech. Des., vol. 131(01), pp. 011013, 2008.

[7] Chablat D., Jha R., Rouillier F., Moroz G., Workspace and joint space analysis of the 3-RPS parallel robot, In: Proceedings of ASME 2014 International Design Engineering Technical Conferences \& Computers and Information in Engineering Conference, Buffalo, United States, pp. 1-10, 2014.

[8] Jha R., Chablat D., Rouillier F., Moroz G., An Algebraic Method to Check the Singularity-Free Paths for Parallel Robots, In: Proceedings of ASME 2015 International Design Engineering Technical Conferences \& Computers and Information in Engineering Conference, Boston, Massachusetts, USA, Vol. 5C, pp. 1-10, 2015.

[9] Pashkevich A., Chablat D., Wenger P., Kinematic calibration of Orthoglide-type mechanisms from observation of parallel leg motions, Mechatronics, Volume 19(04), pp. 478-488, 2009. 